\section{Aceptación docente de las tecnologías digitales en la educación superior: Evolución y uso desde los modelos y las teorías que la explican.}

\author{
Byron Geovanny Hidalgo-Cajo \\ Rebut: 18/06/2020 Acceptat: 20/07/2020
}

\title{
Resumen
}

En la actualidad, es necesario considerar la problemática que se desprende de la sociedad digital y las transformaciones que está viviendo el profesorado universitario para adaptarse a los cambios generados por las tecnologías digitales (TD), en consecuencia, emerge la necesidad de analizar el contexto en el que están ocurriendo estos cambios, y se impulsa la idea de encontrar teorías que ayuden a interpretar y a reflexionar sobre temas relacionados con la tecnología y la educación. Para conocer el estado en cuestión de la aplicabilidad de las teorías y modelos de la adopción tecnológica en el profesorado universitario y lograr revelar cuales son los constructos más sobresalientes y utilizados, se procedió a la revisión sistemática de la literatura de los modelos y teorías de adopción tecnológica permitiéndonos dar luz a los avances en este campo, es así que se recopilaron 321 documentos y mediante criterios de inclusión, exclusión y calidad, se seleccionaron 47 artículos estrictamente relacionados al análisis. Por un lado se ha demostrado que a partir de la teoría de la Difusión de las Innovaciones (TDI), han surgido diversos modelos y teorías importantes como el modelo de aceptación tecnológica (TAM) y la teoría de la Acción Razonada (TRA), destacándose el modelo TAM como el más robusto y popular, esto ha dado lugar a que sea modificado en varios estudios con la incorporación de nuevos constructos, así pues del TAM original se ha identificado que los constructos más utilizados son: utilidad percibida, la facilidad de uso percibido y la intención conductual de uso, a estos se añaden con mayor frecuencia la norma subjetiva, condiciones facilitadoras y autoeficacia; por otra parte es evidente determinar que la adopción tecnológica en el ámbito educativo también sea analizada desde otra perspectiva como es el pedagógico.

Palabras claves: Adopción tecnológica; modelos; Tecnologías Digitales; Educación Superior; Revision Sistemática de la Literatura.

\begin{abstract}
At present, it is necessary to consider the problems that arise from the digital society and the transformations that university professors are undergoing to adapt to the changes generated by digital technologies (DT). Consequently, the need to analyze the context in which these changes are occurring emerges, and the idea of finding theories that help to interpret and reflect on issues related to technology and education is promoted. In order to know the state in question of the applicability of the theories and models of technology adoption in the university faculty and to be able to reveal which are
\end{abstract}


the most outstanding and used constructs, we proceeded to the systematic review of the literature on models and theories of technology adoption allowing us to shed light on the advances in this field. Thus, 321 documents were compiled and through criteria of inclusion, exclusion and quality, 47 articles strictly related to the analysis were selected. On the one hand, it has been demonstrated that from the theory of Diffusion of Innovations (TDI), several important models and theories have emerged such as the model of technological acceptance (TAM) and the theory of Reasoned Action (TRA), highlighting the TAM model as the most robust and popular, this has led to its modification in several studies with the incorporation of new constructs, so from the original TAM it has been identified that the most used constructs are perceived utility, perceived ease of use and behavioral intent of use, to these are added more frequently the subjective norm, facilitating conditions and self-efficacy; on the other hand it is evident to determine that the technological adoption in the educational field is also analyzed from another perspective such as the pedagogical one.

Key words: Technology adoption; models; digital technologies; higher education; Systematic Literature Review.

\section{Introducción}

El uso de las tecnologías digitales ha alterado las actividades cotidianas de la humanidad (Keane, Keane, \& Blicblau, 2016), no sólo en la vida diaria sino también en los ámbitos del aprendizaje, la enseñanza y la investigación (Atiquil Islam, 2016).

La presencia de las TD en todas las organizaciones y en la educación superior han tenido un desarrollo sorprendente, en la actualidad y se han convertido en uno de los elementos más destacados y característicos de nuestra sociedad entrando, inevitablemente, a establecer y determinar la realidad educativa actual.

La Universidad, como institución de educación superior es la encargada de la formación de los futuros profesionales, y la misma, no debe quedarse al margen de la incorporación de estas tecnologías debiendo, incluso, ser precursora e innovadora de esta adopción mediante la puesta en marcha de mecanismos que la promuevan y la garanticen.

Ineludiblemente nos hallamos ante un nuevo escenario que necesita de modernas formas de abordarlo, convirtiéndoles en este sentido, a los profesores universitarios en un elemento clave para el tan proclamado cambio. Pensar en los docentes universitarios como elemento de cambio implica reflexionar sobre aquellos aspectos que establecen lo que concebimos por un buen docente. Entre estos elementos, se perfilan como una de las competencias básicas del profesorado para el desempeño de su profesión que es necesario que los docentes — sepan hacer - en relación con el uso de las tecnologías para la docencia.

Se puede comprender que la adopción de las TD por parte del profesorado podrá lograr desarrollar competencias que establezcan un buen desempeño docente, identificado el perfil de adopción de las TD, esta información relevante guiará a generar propuestas de mejora orientadas a la calidad de la docencia.

Muchos estudios en el tiempo han logrado identificar variables o constructor en el campo de la tecnología educativa con el fin de facilitar o inhiben la aceptación, uso y la adopción de las tecnologías digitales por parte de los docentes (Becker et al., 2017). Otros estudios demuestran que las TD pueden ser utilizadas para mejorar la enseñanza de los profesores y favorecer el rendimiento académico de los estudiantes (Davies, Mullan, \& Feldman, 2017; Sung, Chang, \& Liu, 2016). 
Desde esta perspectiva la investigación se enmarca en un análisis profundo de los principales modelos y teorías de adopción tecnológica que han surgido en el tiempo, cada uno con diferentes conjuntos de determinantes de adopción, por consiguiente se revisa la literatura con respecto a las adopción de las TD y se discute los modelos y sus extensiones prominentes, de manera que la investigación se enmarca en el objetivo de abordar y revelar con claridad los diferentes constructos o variables que determinan la decisión de la adopción de la tecnología analizando los diversos modelos y/o teorías de adopción tecnológica.

De tal manera que se formula las siguientes preguntas de investigación:

PM1. ¿Cómo ha evolucionado las investigaciones con referencia a los modelos y teorías de adopción tecnológica a lo largo de los años?

PM2. ¿Cuáles son las variables más destacadas en los diferentes estudios de adopción de la tecnología en el contexto educativo?

\section{Importancia de la adopción de las TD en el proceso enseñanza aprendizaje universitario.}

La educación es un sistema complejo que requiere múltiples perspectivas y niveles de análisis para comprender su contextos, dinámicas e interacciones de los actores, y en particular en lo que respecta a las innovaciones en el entorno educativo (Hidalgo, Rivera, \& Delgadillo, 2019)

Como ya se menciona anteriormente uno de los agentes fundamentales para el éxito de la integración de las TD son los docentes, dado que tienen la última palabra para emplear la metodología de enseñanza que consideren más pertinente. Por lo tanto, conocer los factores que condicionan su decisión de utilizar metodologías de aprendizaje mediados por la tecnología resulta fundamental a la hora de diseñar iniciativas de integración tecnológica exitosas.

Es así como la aplicación de estas tecnologías en el ámbito educativo potencia la comunicación y el aprendizaje autónomo del estudiante, además permite llevar el proceso de enseñanza-aprendizaje fuera de las aulas de clase, haciendo posible de esta manera un aprendizaje de manera ubicua (Traxler, 2009).

Sin embargo, a pesar de una masiva incursión de los dispositivos tecnológicos y su potencial didáctico que poseen, el uso de las tecnologías aplicadas a la educación universitaria es todavía un fenómeno poco común con una baja tasa de adopción. Una de las causas de este problema se encuentra en la poca disposición de los docentes a la incorporación de las TD en el proceso de enseñanza- aprendizaje (Teo, 2012). En efecto los docentes juegan un papel clave en el proceso de adopción tecnológica, sin embargo, a pesar de las ventajas que pueden aportar las TD en el aula, estas continúan siendo inutilizadas.

Por lo tanto, la adopción de la TD por parte del profesorado resulta fundamental, dado que este decide de manera completamente voluntaria la metodología que utiliza para impartir sus clases (Teo, 2015), teniendo la última palabra sobre el uso de estos dispositivos en contextos de educación formal e informal (Sang, Valcke, Braak, \& Tondeur, 2010).

Aunque existe muchos factores que intervienen e influyen en la adopción y uso de la tecnología en la educación, como los profesores, estudiantes, instituciones, infraestructura, políticas, etc., el profesorado juegan un papel importante siendo el eje central para la innovación educativa. Desde esta perspectiva 
surge la necesidad de conocer de mejor manera cuales son las variables más relevantes y utilizadas en los diferentes estudios para la adopción de la tecnología mediante la revisión sistemática de la literatura

\section{Metodología}

La metodología empleada en el estudio es la revisión sistemática de la literatura (SLR) para la búsqueda y selección de los estudios con referencia al tema de investigación cuyo objetivo fundamental es identificar qué se conoce del tema, qué se ha investigado y qué aspectos permanecen desconocidos, para lo cual se sigue las indicaciones de (Kitchenham \& Charters, 2007), de ahí que una vez formuladas las preguntas de la investigación, se procede a definir los criterios de inclusión y exclusión para llevar a cabo el proceso de selección de la literatura, paso seguido se procede a identificar las fuentes y bases de datos científicas pertinentes para la exploración de la información, estableciendo fórmulas de ecuaciones de búsqueda, creando relaciones lógicas de manera clara y precisa de los términos de búsqueda de la información, en consecuencia se obtendrá textos completos que serán seleccionados después del análisis de la calidad científica explorando la heterogeneidad y el sesgo de las publicaciones pertinentes al tema de investigación para finalmente difundir los resultados.

De esta forma a continuación, se procede a detallar los criterios de selección tanto de inclusión y exclusión, bases de datos seleccionadas para realizar la búsqueda, las cadenas de búsqueda empleadas, los criterios seguidos para la selección de los trabajos y las publicaciones que fueron finalmente incluidas al término del proceso.

A partir de las preguntas de investigación se establecieron los siguientes criterios para llevar a cabo el proceso de selección de artículos:

Los trabajos de investigación que cumplan los Criterios de Inclusión son:

- Cl.1. Los relacionados con los modelos de aceptación tecnológica.

- Cl3. Los modelos y teorías aplicados en el contexto de la educación formal e informal.

- Cl4. Los artículos publicados en el idioma inglés.

De la misma manera se aplica los Criterios de exclusión a los trabajos:

- CE1. Que no están relacionados con los modelos de aceptación tecnológica.

- CE3. Que no aplican Modelos y teorías en contextos de educación formal e informal.

- CE4. Que no están publicados en el idioma inglés.

Una vez definidos los criterios de selección de las investigaciones se llevó acabo la selección de las bases de datos en donde se realizará la búsqueda de la información estableciendo los siguientes requisitos permisibles en las bases de datos seleccionadas:

- Expresiones lógicas para realizar el proceso de búsqueda.

- Búsquedas en todo el texto de los trabajos o en campos específicos.

- El acceso a los artículos a través de cuentas institucionales o suscripciones personales. 
- Estudios relevantes de contrastada calidad.

Teniendo en cuenta estos criterios se seleccionaron las bases de datos electrónicas SCOPUS y Web of Science (WoS).

De ahí que a partir de las preguntas de investigación y los criterios de inclusión se formularon dos cadenas de búsqueda tanto para SCOPUS como WoS.

En SCOPUS se utilizó la cadena de búsqueda:

TITLE-ABS-KEY ("technology acceptance models and theories")

TITLE-ABS-KEY ("technology acceptance models and theories" AND "teachers university")

De esta manera en WoS se emplearon los mismos términos, adaptando los operadores booleanos a las características del sistema de búsqueda de la base de datos, empleándose finalmente la siguiente cadena:

TS=("technology acceptance models and theories" )

TS=("technology acceptance models and theories" AND teachers university)

El proceso de selección de las publicaciones se llevó a cabo, por última vez, en febrero de 2020 dividido en tres fases. Durante la primera fase, se recopilaron los resultados de búsqueda de los dos repositorios y se introdujeron en una hoja de cálculo (Excel) en la que se fue registrando todo el proceso de selección. Una vez registrados todos los documentos encontrados en los dos repositorios, se eliminaron los elementos duplicados como paso previo a aplicar los criterios de exclusión e inclusión. En esta fase se obtuvieron un total de 321 documentos, 173 en SCOPUS y 148 en WoS. Tras la eliminación de los duplicados el número final de artículos que pasaron a la segunda fase se redujo a 210.

En la segunda fase, se aplicaron los criterios de exclusión e inclusión a través de la consulta del título y el resumen de las publicaciones. En caso de que estos no fueran suficiente para poder tomar una decisión, se optó por dejar las publicaciones pasar a la siguiente fase donde se examinaría el texto completo del artículo en profundidad. En esta fase se descartaron 102 documentos, pasando 108 a la fase siguiente.

Finalmente, en la fase tres se aplicaron los criterios de calidad, donde se examina el texto completo del articulo y se procede a eliminar aquellas publicaciones que pasaron de la fase II debido a la ambigüedad de su título y resumen, quedando 47 artículos seleccionados que cumplen con la profundidad de la información para responder a las preguntas de investigación (Figura 1). 


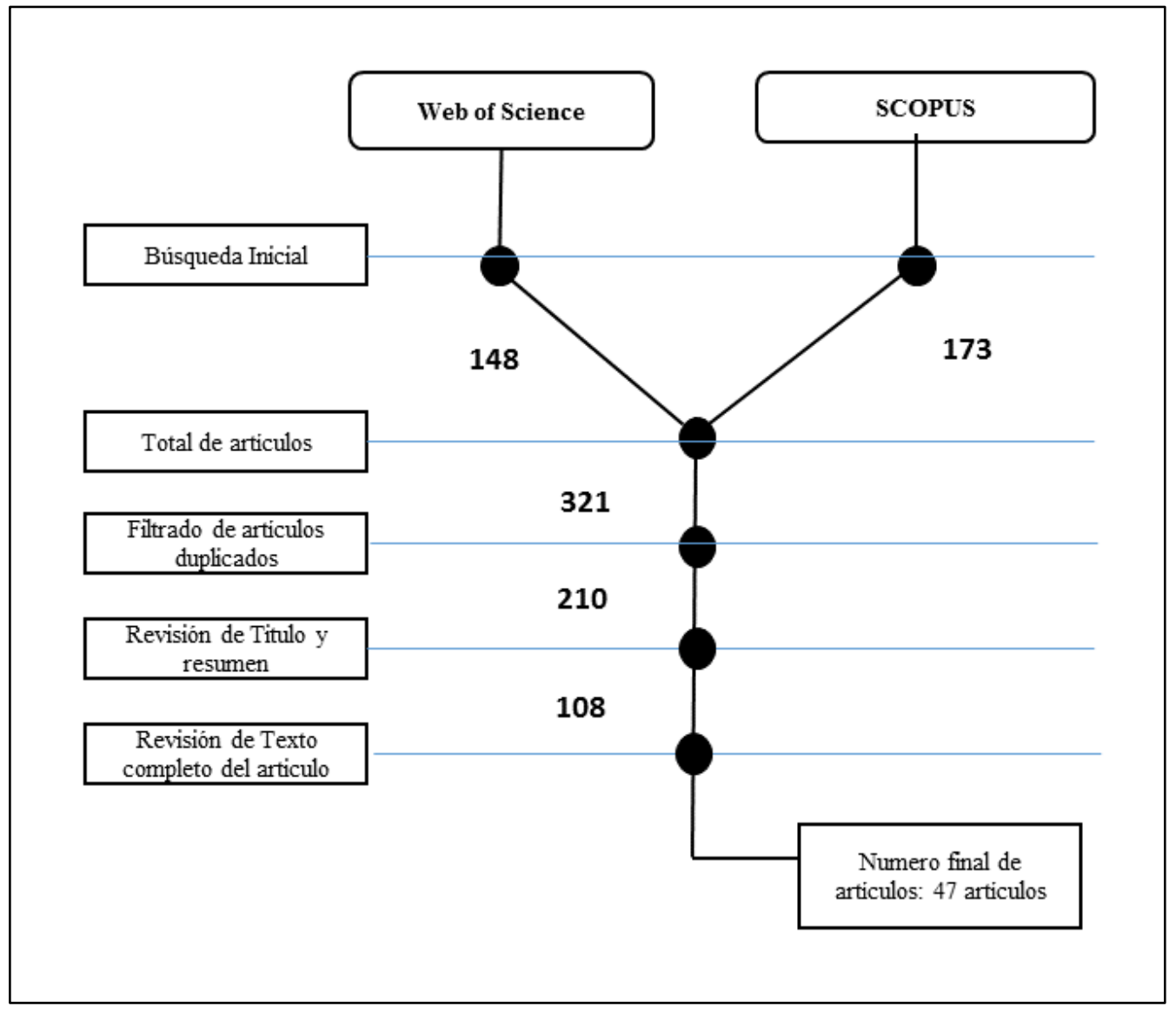

Figura 1.Proceso de selección.

\section{Resultados}

Se inicia esta apartado pretendiendo responder a la primera pregunta de la investigación que está centrada en las tendencias más importantes en cuanto al diseño de modelos y teorías de adopción tecnológica a lo largo de los años.

Se puede observar la evolución y el gran desarrollo de investigaciones sobre la aceptación de la tecnología en el ámbito educativo, estos estudios principalmente emergen de los modelos referentes a la adopción de la tecnología, tal como el modelo de aceptación de la tecnología (TAM), así como la teoría de la acción razonada (TRA), la teoría planeada del comportamiento, difusión de la innovación, UTAUT, entre otros, que se detalla a continuación. Algunos de los modelos mencionados anteriormente se originan en la teoría de la innovación tecnológica (Moore \& Benbasat, 1991; Rogers, 1995), mientras otros se centraron en factores internos (subjetivos) (Ajzen, 1991a; F. Davis, 1989).

El modelo TAM original se ha ido desarrollando y creciendo en complejidad, incorporando nuevas variables que enriquecen y se generan nuevos TAM extendidos, al respecto se puede mencionar algunas de las evoluciones más importantes como son el TAM 2 (Venkatesh \& Davis, 2000), TAM 3 (Venkatesh \& Bala, 2008) , la Teoría Unificada de la Aceptación y el uso de la tecnología (UTAUT) (Venkatesh, Morris, Davis \& Davis, 2003).

Al referirnos a las teorías de adopción tecnológica en la Tabla 1 se presenta las principales teorías que han dado lugar a la generación de nuevas teorías y modelos de adopción tecnológica. 
Tabla 1: Teorías de la adopción tecnológica individual.

\begin{tabular}{|c|c|}
\hline TEORIA & Variables Básicas \\
\hline Teoría de la Acción Razonada (TRA) (Ajzen, 1975) & $\begin{array}{l}\text { Actitud hacia la Conducta/comportamiento; Norma } \\
\text { Subjetiva }\end{array}$ \\
\hline $\begin{array}{l}\text { Teoría del Comportamiento Planeado (TPB) (Taylor \& } \\
\text { Todd, 1995) }\end{array}$ & $\begin{array}{l}\text { Actitud hacia el Comportamiento; Norma subjetiva; } \\
\text { Control del comportamiento percibido }\end{array}$ \\
\hline Teoría de la Difusión de la innovación (TDI) (Rogers, 1962) & $\begin{array}{l}\text { Ventaja Relativa; Facilidad de Uso; Imagen; Viabilidad; } \\
\text { Compatibilidad; Resultados Demostración; Voluntariedad } \\
\text { de uso }\end{array}$ \\
\hline Teoría Cognitiva Social (SCT) (Bandura, 1986) & $\begin{array}{l}\text { Rendimiento de las expectativas de resultados; } \\
\text { Expectativas de resultados personales; Autoeficacia; } \\
\text { Afectar; Ansiedad }\end{array}$ \\
\hline $\begin{array}{l}\text { Teoría Unificada de Aceptación y Uso de la tecnología } \\
\text { (UTAUT). (Venkatesh, Morris, Davis, \& Davis, 2003) }\end{array}$ & $\begin{array}{l}\text { Voluntariedad de uso; Experiencia; Edad; Genero; } \\
\text { Condiciones facilitadoras; Influencia social; Expectativa de } \\
\text { esfuerzo; Expectativa de rendimiento }\end{array}$ \\
\hline
\end{tabular}

El análisis que hemos realizado manifiesta que, a partir de los planteamientos de Rogers, surgen distintas corrientes, modelos y teorías que buscan descubrir inexactitudes detectadas en el TDI, con el fin de dar una posible solución a esta debilidad. Una de estas inexactitudes detectadas está relacionada con la tendencia del TDI a comprender los fenómenos pasados y no hacia la posibilidad de predecir la adopción futura (Hsu, Lu, \& Hsu, 2007). Como respuesta a esta necesidad emergen diversos modelos como se aprecia en la tabla 2, entre ellos el Modelo de adopción de la tecnología (TAM) y la Teoría de la Acción Razonada (TRA), los mismos que adoptan un subconjunto de características del trabajo de Rogers y agregan específicamente la variable actitud con respecto a la innovación.

Tabla 2: Modelos de la adopción tecnológica individual.

\begin{tabular}{|c|c|}
\hline MODELO & Constructos Básicos \\
\hline $\begin{array}{l}\text { Modelo de adopción de la tecnología (TAM) (F. Davis, } \\
\text { 1989) }\end{array}$ & $\begin{array}{l}\text { Utilidad Percibida; Facilidad de Uso Percibida, Actitud } \\
\text { hacia el uso }\end{array}$ \\
\hline $\begin{array}{l}\text { Modelo Motivacional (MM) (F. D. Davis, Bagozzi, \& } \\
\text { Warshaw, 1992; Vallerand, 1997; Venkatesh et al., 2003) }\end{array}$ & Motivación extrínseca; Motivación intrínseca \\
\hline $\begin{array}{l}\text { TAM y TPB combinados (C-TAM-TPB) (Taylor \& Todd, } \\
\text { 1995) }\end{array}$ & $\begin{array}{l}\text { Actitud hacia el Comportamiento; Norma subjetiva; } \\
\text { Control del comportamiento percibido; Utilidad Percibida }\end{array}$ \\
\hline $\begin{array}{l}\text { Modelo de Utilización de PC (MPCU) (Triandis, 1977; } \\
\text { Thompson, Higgins, \& Howell, 1991) }\end{array}$ & $\begin{array}{l}\text { Ajuste de Trabajo; Complejidad; Consecuencias a largo } \\
\text { plazo; Afecto hacia el uso; Factores Sociales; Condiciones } \\
\text { facilitadoras }\end{array}$ \\
\hline
\end{tabular}

Como consecuencia de lo anterior el modelo TAM se destaca y goza de mayor reconocimiento manteniendo su notoriedad por su robustez, parsimonia y poder explicativo (Y. Liu, Li, \& Carlsson, 2010), 
siendo uno de los modelos preferidos y validos en la exploración de los comportamientos de adopción de la tecnología por parte de los usuarios, cuyo objetivo es predecir las intenciones del comportamiento y uso de las tecnologías (Lemay, Morin, Bazelais, \& Doleck, 2018), este modelo ha estado bajo un análisis y exploración continua por parte de los investigadores desde diversas perspectivas.

Davis (1989) estableció que "la utilidad percibida y la facilidad de uso representan las convicciones que llevan a la aceptación de la tecnología y son parte esencial del modelo" (p. 319). Es así que, durante muchos años, entre investigadores y profesionales, ha existido una gran preocupación acerca de asegurar la adopción exitosa de tecnologías dentro de las organizaciones. Un modelo clave para lograr este objetivo ha sido TAM, siendo el más probado y validado de todos los existentes. "TAM responde efectivamente a una necesidad de poder entender las motivaciones de los usuarios al adoptar una tecnología y predecir" (Cataldo, 2013, p. 1).

El modelo TAM dado su notoriedad ha sido analizado y estudiado por diferentes autores (Al-Emran, Mezhuyev, \& Kamaludin, 2018; Hsu et al., 2007; Nagy, 2018; Venkatesh \& Davis, 2000) por su fuerte tendencia en la generación de modelos extendidos, es así que se destacan la gran heterogeneidad de las propuestas de investigación, las cuales aplican en un menor grado el TAM original, frente a una gran mayoría de los TAM extendidos.

Es así que existe numerosas variables incluidas en los TAM extendidos, de manera que los modelos TAM 2 y TAM 3 son los más dominantes. EI TAM 2 incluye los constructos como utilidad percibida, intenciones de uso, norma subjetiva, voluntariedad, imagen, relevancia laboral, calidad de los resultados, demostrabilidad de los resultados y facilidad de uso percibida. EI TAM 3 está compuesto por: utilidad percibida, la facilidad de uso percibida, la adopción y el uso de la tecnología. (Yu, 2020).

A favor de lo expresado en los últimos años, los investigadores han adaptado algunas de estas teorías para investigar su capacidad de comprender la aceptación de la tecnología por parte del profesorado, mediante los diferentes estudios realizados tanto en el TAM como en los TAM extendidos, ¿es así que surge la motivación de analizar cuáles son las variables más destacadas y aplicadas del modelo TAM en el contexto educativo?. Con el objetivo de dar respuesta a esta interrogante se logra recopilar estudios basados en este modelo como se puede apreciar en la tabla 3.

Tabla 3: Investigaciones TAM y TAM expandidos

\begin{tabular}{|c|c|c|c|c|c|c|c|c|c|c|c|c|c|c|}
\hline Autor/es & TAM & TAM-E & PU & PEOU & AT & BI & AE & CF & NS & SP & IP & EP & DP & NP \\
\hline \multicolumn{15}{|l|}{ Luan \& Teo } \\
\hline (2009) & $x$ & & $x$ & $x$ & $x$ & $x$ & & & & & & & & \\
\hline Timothy Teo (2010) & & $x$ & $x$ & $x$ & $x$ & $x$ & & $x$ & $x$ & & & & & \\
\hline $\begin{array}{l}\text { I. F. Liu, Chen, Sun, } \\
\text { Wible, \& Kuo ( 2010) }\end{array}$ & & $x$ & $x$ & $x$ & & $x$ & & & & & $x$ & $\mathrm{x}$ & & \\
\hline $\begin{array}{l}\text { Timothy Teo, Ursavaş, } \\
\text { \& Bahçekapili (2011) }\end{array}$ & $\mathrm{x}$ & & $\mathrm{x}$ & $x$ & $x$ & $\mathrm{x}$ & & & & & & & & \\
\hline $\begin{array}{l}\text { T Teo, Ursavaş, \& } \\
\text { Bahçekapili (2012) }\end{array}$ & & $x$ & $x$ & $x$ & $x$ & $x$ & & $x$ & $x$ & & & & & \\
\hline $\begin{array}{l}\text { Tarcan, Varol, Kantarci, } \\
\text { \& Firlar (2012) }\end{array}$ & & $x$ & $x$ & $\mathrm{x}$ & $x$ & $x$ & & $x$ & $x$ & & & & & \\
\hline
\end{tabular}




\begin{tabular}{|c|c|c|c|c|c|c|c|c|c|c|c|c|c|}
\hline Timothy Teo (2012) & $x$ & $x$ & $x$ & $x$ & $\mathrm{x}$ & & $x$ & $\mathrm{x}$ & & & & & \\
\hline $\begin{array}{l}\text { Wong, Teo, \& Russo } \\
\text { (2012) }\end{array}$ & $x$ & $x$ & $x$ & & $x$ & $x$ & & & & & & & \\
\hline $\begin{array}{l}\text { Motaghian, } \\
\text { Hassanzadeh, \& } \\
\text { Moghadam (2013) }\end{array}$ & $x$ & $\mathrm{x}$ & $x$ & & $\mathrm{x}$ & $x$ & & $x$ & & & & & \\
\hline Islam (2014) & $x$ & $x$ & $x$ & & & $x$ & & & $x$ & & & & \\
\hline Ros et al.(2015) & $x$ & $x$ & $x$ & & $x$ & & & & & $x$ & $x$ & & \\
\hline Timothy Teo (2015) & $x$ & $x$ & $x$ & $x$ & & & & $x$ & & & & & \\
\hline $\begin{array}{l}\text { Basak, Gumussoy, \& } \\
\text { Calisir (2015) }\end{array}$ & $x$ & $x$ & $x$ & & $\mathrm{x}$ & $x$ & & $x$ & & & & $x$ & $x$ \\
\hline $\begin{array}{l}\text { Okyere-Kwakye, Nor, } \\
\text { \& Ologbo (2016) }\end{array}$ & $x$ & $x$ & $x$ & $x$ & $x$ & $x$ & & & & & & $x$ & \\
\hline (Park \& Kwon (2016) & $X$ & $x$ & $x$ & $X$ & $X$ & & & & & & & $x$ & \\
\hline $\begin{array}{l}\text { Sánchez-Mena, Martí- } \\
\text { Parreño, \& Aldás- } \\
\text { Manzano (2017) }\end{array}$ & $x$ & $x$ & $x$ & $x$ & $x$ & $x$ & & $x$ & & & & $x$ & \\
\hline Fokides ( 2017) & $x$ & $x$ & $x$ & $\mathrm{x}$ & $\mathrm{x}$ & $x$ & & & & & & & \\
\hline Jeong \& Kim (2017) & $x$ & $x$ & $x$ & & $x$ & $x$ & & $x$ & & & & & $x$ \\
\hline $\begin{array}{l}\text { Al-Azawei, Parslow, \& } \\
\text { Lundqvist ( 2017) }\end{array}$ & $x$ & $x$ & $x$ & & & $x$ & & & $x$ & & & & \\
\hline $\begin{array}{l}\text { Esteban-Millat, } \\
\text { Martínez-López, Pujol- } \\
\text { Jover, Gázquez-Abad, } \\
\text { \& Alegret (2018) }\end{array}$ & $x$ & $x$ & $x$ & $x$ & $x$ & & & & & & & & \\
\hline Lemay et al. (2018) & $x$ & $x$ & $x$ & $x$ & $x$ & $x$ & $x$ & $x$ & & & & & \\
\hline Mazman Akar (2019) & $x$ & $x$ & $x$ & $x$ & $x$ & & & $x$ & & & & & $x$ \\
\hline $\begin{array}{l}\text { Bin, Islam, Gu, Spector, } \\
\& \text { Wang }(2020)\end{array}$ & $x$ & $x$ & $x$ & & $x$ & $x$ & & & & & & & \\
\hline
\end{tabular}

Nota: Utilidad Percibida (PU), Facilidad de uso percibida (PEOU), Actitud de uso (AT), Intensión Conductual (BI), Autoeficacia (AE), Condiciones facilitantes (CF), Norma subjetiva (NS), Satisfacción percibida(S), Interacción percibida (IP), Experiencia previa (EP), Disfrute percibido (DP), Innovación personal (NP).

Al hablar de las dificultades que pasa la adopción de la tecnológica por parte del profesorado y su aplicación en el proceso educativo con el fin de conseguir la calidad en el proceso enseñanza aprendizaje, este va a variar según el momento, es decir a las situaciones, necesidades, intereses y convicciones del entorno donde se desarrolla (Hidalgo Cajo, 2015). 
Actualmente los estudios sobre el modelo de aceptación tecnología (TAM) original, parece ser menos numerosos, es así como mediante la revisión sistemática de la literatura se demuestra que cada vez existen más inclinación a modificar o añadir más constructos al TAM y enfocarse al análisis de los TAM extendidos.

A tal efecto al examinar la aceptación de la tecnología de los individuos es una forma de determinar la intención del profesor hacia el uso de tecnologías en su práctica educativa (Scherer, Siddiq, \& Tondeur, 2019). A tal fin se puede evidenciar en los diferentes estudios que dan a conocer que el modelo TAM, y en especial el Extendido, se ha aplicado ampliamente en determinar la adopción de la tecnología por parte del profesorado, La mayoría de los estudios analizados son validados por medio del método de ecuaciones estructurales basados en covarianza (CB-SEM) y otros en menor escala, aplican el método mínimos cuadrados parciales (PLS-SEM

El análisis pudo detectar que los constructos más utilizados del TAM original son: la utilidad percibida, la facilidad de uso percibido y la intención conductual de uso, de la misma manera el análisis revela los constructos que se integran con más frecuencia al TAM original dando lugar a los TAM extendidos como son: norma subjetiva, condiciones facilitadoras y autoeficacia, desde esta perspectiva actual se constata la existencia de un creciente interés en la aplicación de modelos basados en TAM extendidos para analizar la adopción tecnológica de los docentes,

\section{Discusión}

Después del análisis de la información, es de gran utilidad discutir los resultados obtenidos, uno de estos identifica al modelo de adopción tecnológica TAM como el más utilizado y aplicado para medir el nivel de aceptación de la tecnología, el mismo que ha atraído una atención significativa en investigaciones de diversos autores tales como (Liaw, 2008; Shin \& Kang, 2015; Sun y otros, 2008; Teng, 2015; Teo, 2008). La investigación coincide con estudios realizados por Kocaleva, Stojanovic, \& Zdravev (2015); I. F. Liu et al. (2010) quienes manifiesta que los modelos TAM y TAM extendido son los más populares y utilizados en valorar la adopción tecnológica. Por otra parte, autores como Venkatesh y Davis (1996) iniciaron con la idea de que el modelo puede ampliarse para aumentar su eficacia, así pues se evidencia que desde el modelo TAM existe una gran expansión de modelos que integran nuevas variables de otros modelos, que en conjunto forman los TAM extendidos, desde esta perspectiva estudios realizados por Legris, Ingham y Collerette (2003) examinaron críticamente el TAM, y afirmaron que se deberían incluir otras variables para comprender los factores que afectan a la adopción de la tecnología.

Otro punto importante fue analizar cuáles son las variables más destacadas en los diferentes estudios de adopción de la tecnología en el contexto educativo, tal como se pudo identificar anteriormente se conoce que el TAM y en especial los TAM extendidos son los más utilizados en el análisis de adopción de la tecnología, como resultado de los expresado se puede mencionar que las variables del TAM más aplicadas en los diferentes estudios son: utilidad percibida, la facilidad de uso percibido e intención conductual de uso, este planteamiento es congruente con el estudio de Islam (2014), y Davis (1989), de igual modo (Kang \& Shin, 2015), indica -según el modelo formulado por Davis, Bagozzi \& Warshaw (1992)- que estas variables son claras predictoras y determinantes de la utilización que hagan de las tecnologías tanto docentes (Teo, 2010, 2012) como estudiantes (Vera, Torres y Martínez, 2014). Sin embargo, esta conclusión fue refutada por Edmunds, Thorpe y Conole (2012) cuando manifiesta que los dos factores de la TAM (utilidad percibida y facilidad de uso percibido) pueden no identificar todos los componentes significativos para predecir la aceptación de la tecnología. Además, uno de los debates sobre la TAM es que no tuvo en cuenta las diferencias de usuarios y culturas, ni su influencia en la adopción de la tecnología (Bagozzi 2007; Benbasat y Barki 2007; Straub y Burton-Jones 2007, por otro lado, dado los diversos tipos de TAM extendidos que añaden varios constructos, es necesario y significativo determinar los constructos más frecuentes que han sido aplicados en las investigaciones 
siendo: la norma subjetiva, condiciones facilitadoras y autoeficacia resultados corroborados con estudios realizados por (Islam, 2014).

El estudio ha permitido mejorar la capacidad predictiva del modelo TAM original, así como complementarlo con variables que ofrecen una mejor comprensión de la adopción de la tecnología.

\section{Conclusiones}

La tecnología digital se utiliza cada vez más en la educación y su integración en el proceso educativo ha tenido una enorme importancia en el fomento de la educación basada en la tecnología.

Es así que cada vez se hace necesario analizar los diferentes factores que influyen en la adopción de la tecnología, a tal efecto se hace imperante examinar modelos que ayuden a dar luz a la integración de las TD en el ámbito educativo, la revisión de la literatura da inicio desde la teoría TDI que es una de las teorías más ampliamente utilizadas y aceptadas al momento de analizar, entender y explicar el fenómeno de la adopción de la innovación, especialmente en la disciplina de sistemas de información.

Entre las características destacadas del TDI se tiene la Ventaja Relativa, Compatibilidad y la complejidad, es así como los modelos principales que han nacido de los planteamientos de Rogers se destacan la TRA y TAM, que retoman algunas de las características, modificándolas y redefiniéndolas de acuerdo con las necesidades propias de cada autor.

En los últimos años, los investigadores han adaptado algunas de estas teorías para investigar su capacidad de comprender la aceptación de la tecnología por parte del profesorado, siendo el modelo TAM el más utilizado, a partir de entonces los resultados de la investigación confirman que el TAM predice con éxito el comportamiento de los usuarios en la adopción de la tecnología.

El modelo TAM ha tenido una atención significativa en la investigación y ha sido analizado desde diferentes perspectivas, a tal punto que del TAM original han surgido los TAM expandidos donde se incluyen constructos de otras teorías/modelos o nuevos constructos.

No obstante, los estudios sobre el TAM original parecen ser relativamente menos numerosos, no siendo así los TAM extendidos, que han sido analizados ampliamente con respecto a la adopción de la tecnología en el entorno educativo, además se detecta que la gran mayoría de estudios fueron validados mediante la utilización de modelos de ecuaciones estructurales basados en covarianza (CB-SEM) hallazgo paralelo a los estudios de Sánchez-Mena et al, (2017).

La investigación identifica los constructos más utilizados del TAM como son: utilidad percibida, la facilidad de uso percibido, intención conductual de uso, por otro lado, los constructos más frecuentes que se incluyen en el TAM expandido son: norma subjetiva, condiciones facilitadoras y autoeficacia.

En consecuencia, dados los diversos tipos de TAM extendidos que añaden numerosos constructos, es necesario y significativo determinar el impacto y las estructuras de conocimiento de los modelos extendidos. Es así que la revisión de la literatura sobre los modelos extendidos puede preparar un terreno sólido para futuras investigaciones.

Igualmente, al evaluar en nivel de apropiación de la tecnología de los usuarios no es suficiente con conocer el grado de satisfacción con respecto a la tecnología, además, es necesario conocer para que utilizan las TD, cuál es su opinión sobre la tecnología y cuáles son las características de los recursos y los dispositivos que usan. El hecho de tener todos estos aspectos identificados nos permitirá determinar los 
conocimientos y habilidades que poseen los usuarios, con el fin de formular estrategias que permitan su desarrollo.

\section{Referencias Bibliográficas}

Ajzen, I. (1991a). Measuring oral health behaviour in Flemish health care workers: An application of the theory of planned behaviour. Community Dental Health, 25(2), 107-114. Retrieved from: https://doi.org/10.1922/CDH_2120VandenBroucke08

Al-Azawei, A., Parslow, P., \& Lundqvist, K. (2017). Investigating the effect of learning styles in a blended e-learning system: An extension of the technology acceptance model (TAM). Australasian Journal of Educational Technology, 33(2), 1-23. Retrieved from: https://doi.org/10.14742/ajet.2741

Al-Emran, M., Mezhuyev, V., \& Kamaludin, A. (2018). Technology Acceptance Model in M-learning context: A systematic review. Computers and Education, 125, 389-412. Retrieved from: https://doi.org/10.1016/j.compedu.2018.06.008

Atiquil Islam, A. Y. M. (2016). Development and validation of the technology adoption and gratification (TAG) model in higher education: A cross-cultural study between Malaysia and China. International Journal of Technology and Human Interaction, 12(3), 78-105. Retrieved from: https://doi.org/10.4018/IJTHI.2016070106

Bandura, A. (1986). Social foundations of thought and action. Englewood Cliffs, NJ, 1986.

Basak, E., Gumussoy, C. A., \& Calisir, F. (2015). Examining the factors affecting PDA acceptance among physicians: An extended technology acceptance model. Journal of Healthcare Engineering, 6(3), 399-418. Retrieved from: https://doi.org/10.1260/2040-2295.6.3.399

Becker, S. A., Cummins, M., Davis, A., Freeman, A., Hall, C. G., \& Ananthanarayanan, V. (2017). NMC horizon report: 2017 higher education edition. Retrieved from The New Media Consortium:

Bin, E., Islam, A. Y. M. A., Gu, X., Spector, J. M., \& Wang, F. L. (2020). A study of Chinese technical and vocational college teachers' adoption and gratification in new technologies. British Journal of Educational Technology, O(0). Retrieved from: https://doi.org/10.1111/bjet.12915

Davies, S., Mullan, J., \& Feldman, P. (2017). Rebooting learning for the digital age: What next for technology-enhanced higher education? HEPI Report, 93, 46. Retrieved from: http://www.hepi.ac.uk/wp-content/uploads/2017/02/Hepi_Rebooting-learning-for-the-digitalage-Report-93-02_02_17Web.pdf

Davis, F. (1989). Perceided Usefulness, Perceived Ease of Use, and User Acceptance of Information Technology. 15th World Conference on Earthquake Engineering (15WCEE), 13(3), 319-340. Retrieved from: https://doi.org/10.1016/S0305-0483(98)00028-0

Davis, F. D., Bagozzi, R. P., \& Warshaw, P. R. (1992). Extrinsic and intrinsic motivation to use computers in the workplace. Journal of Applied Social Psychology, 22(14), 1111-1132. 
Esteban-Millat, I., Martínez-López, F. J., Pujol-Jover, M., Gázquez-Abad, J. C., \& Alegret, A. (2018). An extension of the technology acceptance model for online learning environments. Interactive Learning Environments, 26(7), 895-910. Retrieved from: https://doi.org/10.1080/10494820.2017.1421560

Fokides, E. (2017). Pre-service teachers' intention to use MUVES as practitioners - A structural equation modeling approach. Journal of Information Technology Education: Research, 16(1), 47-68. Retrieved from: https://doi.org/10.28945/3645

Hidalgo, B. G., Rivera, L., \& Delgadillo, R. (2019). Integration of Learning Management System Technology and Social Networking Sites in the E-Learning Mode: A Review and Discussion. ASEE Computers in Education (COED)Journal, 10(2), 1-13. Retrieved from: http://aseecoed.org/index.php/coed/article/view/418

Hidalgo Cajo, B. G. (2015). Analisis Matematico del modelo educativo usando facebook: caso de estudio. Universidad Nacional de Chimborazo. Retrieved from: http://openaccess.uoc.edu/webapps/o2/handle/10609/43111

Hsu, C. L., Lu, H. P., \& Hsu, H. H. (2007). Adoption of the mobile Internet: An empirical study of multimedia message service (MMS). Omega, 35(6), 715-726. Retrieved from: https://doi.org/10.1016/j.omega.2006.03.005

Islam, A. Y. M. A. (2014). Validation of the technology satisfaction model (TSM) Developed in higher education: The application of structural equation modeling. International Journal of Technology and Human Interaction, 10(3), 44-57. Retrieved from; https://doi.org/10.4018/ijthi.2014070104

Jeong, H. I., \& Kim, Y. (2017). The acceptance of computer technology by teachers in early childhood education. Interactive Learning Environments, 25(4), 496-512. Retrieved from: https://doi.org/10.1080/10494820.2016.1143376

Keane, T., Keane, W. F., \& Blicblau, A. S. (2016). Beyond traditional literacy: Learning and transformative practices using ICT. Education and Information Technologies, 21(4), 769-781. Retrieved from: https://doi.org/10.1007/s10639-014-9353-5

Kitchenham, B., \& Charters, S. (2007). Guidelines for performing Systematic Literature Reviews in Software Engineering. Technical Report, 2(3). Retrieved from: http://citeseerx.ist.psu.edu/viewdoc/summary?doi=10.1.1.117.471

Kocaleva, M., Stojanovic, I., \& Zdravev, Z. (2015). Model of e-Learning Acceptance and Use for Teaching Staff in Higher Education Institutions. International Journal of Modern Education and Computer Science, 7(4), 23-31. Retrieved from: https://doi.org/10.5815/ijmecs.2015.04.03

Lemay, D. J., Morin, M. M., Bazelais, P., \& Doleck, T. (2018). Modeling Students' Perceptions of SimulationBased Learning Using the Technology Acceptance Model. Clinical Simulation in Nursing, 20, 2837. Retrieved from: https://doi.org/10.1016/j.ecns.2018.04.004

Liu, I. F., Chen, M. C., Sun, Y. S., Wible, D., \& Kuo, C. H. (2010). Extending the TAM model to explore the factors that affect Intention to Use an Online Learning Community. Computers and Education, 54(2), 600-610. Retrieved from: https://doi.org/10.1016/j.compedu.2009.09.009 
Liu, Y., Li, H., \& Carlsson, C. (2010). Factors driving the adoption of m-learning: An empirical study. Computers and Education, 55(3), 1211-1219. Retrieved from: https://doi.org/10.1016/j.compedu.2010.05.018

Luan, W. S., \& Teo, T. (2009). Investigating the Technology Acceptance among Student Teachers in Malaysia: An Application of the Technology Acceptance Model (TAM). The Asia-Pacific Education Researcher, 18(2). Retrieved from: https://doi.org/10.3860/taper.v18i2.1327

Mazman Akar, S. G. (2019). Does it matter being innovative: Teachers' technology acceptance. Education and Information Technologies, 24(6), 3415-3432. Retrieved from: https://doi.org/10.1007/s10639-019-09933-z

Moore, G. C., \& Benbasat, I. (1991). Moore, G. C., \& Benbasat, I. (1991). Development of an instrument to measure the perceptions of adopting an information technology innovation., (January 2018). Retrieved from: https://doi.org/10.1287/isre.2.3.192

Motaghian, H., Hassanzadeh, A., \& Moghadam, D. K. (2013). Factors affecting university instructors' adoption of web-based learning systems: Case study of Iran. Computers and Education, 61(1), 158-167. Retrieved from: https://doi.org/10.1016/j.compedu.2012.09.016

Nagy, J. (2018). Evaluation of Online Video Usage and Learning Satisfaction: An Extension of the Technology Acceptance Model. International Review of Research in Open and Distributed Learning, 19(1).

Okyere-Kwakye, E., Nor, K. M., \& Ologbo, A. C. (2016). Technology acceptance: Examining the intentions of ghanaian teachers to use computer for teaching. African Journal of Library Archives and Information Science, 26(2), 119-132.

Park, E., \& Kwon, S. J. (2016). The adoption of teaching assistant robots: A technology acceptance model approach. Program-Electronic Library and Information System., 50(4), 354-366. Retrieved from: https://doi.org/10.1108/PROG-02-2016- 0017

Renda dos Santos, L. M., \& Okazaki, S. (2014). Marco Teórico de la Adopción de la Enseñanza On-Line. Desafio Online, 2, 790-813. Retrieved from: https://www.bps.go.id/dynamictable/2018/05/18/1337/persentase-panjang-jalan-tol-yangberoperasi-menurut-operatornya-2014.html

Rogers, E. M. (1962). Diffusion of Innovations. Inc., New York.

Rogers, E. M. (1995). Diffusion of Innovations4 The Free Press New York Google Scholar.

Ros, S., Hernández, R., Caminero, A., Robles, A., Barbero, I., Maciá, A., \& Holgado, F. P. (2015). On the use of extended TAM to assess students' acceptance and intent to use third-generation learning management systems. British Journal of Educational Technology, 46(6), 1250-1271. Retrieved from: https://doi.org/10.1111/bjet.12199

Sánchez-Mena, A., Martí-Parreño, J., \& Aldás-Manzano, J. (2017). The effect of age on teachers' intention to use educational video games: A TAM approach. Electronic Journal of E-Learning, 15(4), 355366. Retrieved from: https://files.eric.ed.gov/fulltext/EJ1154704.pdf 
Sang, G., Valcke, M., Braak, J. van, \& Tondeur, J. (2010). Student teachers' thinking processes and ICT integration: Predictors of prospective teaching behaviors with educational technology. Computers and Education, 54(1), 103-112. Retrieved from: https://doi.org/10.1016/j.compedu.2009.07.010

Scherer, R., Siddiq, F., \& Tondeur, J. (2019). The technology acceptance model (TAM): A meta-analytic structural equation modeling approach to explaining teachers' adoption of digital technology in education. Computers and Education, 128, 13-35. Retrieved from: https://doi.org/10.1016/j.compedu.2018.09.009

Sung, Y. T., Chang, K. E., \& Liu, T. C. (2016). The effects of integrating mobile devices with teaching and learning on students' learning performance: A meta-analysis and research synthesis. Computers and Education, 94, 252-275. Retrieved from: https://doi.org/10.1016/j.compedu.2015.11.008

Tarcan, E., Varol, E. S., Kantarci, K., \& Firlar, T. (2012). A study on Kazakh Academicians' information technology acceptance. Kazak Akademisyenlerin Bilgi Teknolojilerini Kabulü Üzerine Bir Araştidotlessrma, 62(62), 205-230. Retrieved from: http://www.acarindex.com/dosyalar/makale/acarindex-1423873036.pdf

Taylor, S., \& Todd, P. (1995). Understanding information technology usage: A test of competing models. Information Systems Research, 6(October 2014), 144-176. Retrieved from: http://pubsonline.informs.org/doi/pdf/10.1287/isre.6.2.144

Teo, T, Ursavaş, O. F., \& Bahçekapili, E. (2012). An assessment of pre-service teachers' technology acceptance in Turkey: A structural equation modeling approach. Asia-Pacific Education Researcher, 21, 191-202. Retrieved from: http://www.scopus.com/inward/record.url?eid=2-s2.084858763296\&partnerID=40\&md5=ef3aac58ae408c5dcc82c482d62e6f92

Teo, Timothy. (2010). Examining the influence of subjective norm and facilitating conditions on the intention to use technology among pre-service teachers: A structural equation modeling of an extended technology acceptance model. Asia Pacific Education Review, 11(2), 253-262. Retrieved from: https://doi.org/10.1007/s12564-009-9066-4

Teo, Timothy. (2012). Examining the intention to use technology among pre-service teachers: An integration of the Technology Acceptance Model and Theory of Planned Behavior. Interactive Learning Environments, 20(1), 3-18. Retrieved from https://doi.org/10.1080/10494821003714632

Teo, Timothy. (2015). Comparing pre-service and in-service teachers' acceptance of technology: Assessment of measurement invariance and latent mean differences. Computers and Education, 83, 22-31. Retrieved from: https://doi.org/10.1016/j.compedu.2014.11.015

Teo, Timothy, Ursavaş, Ö. F., \& Bahçekapili, E. (2011). Efficiency of the technology acceptance model to explain pre-service teachers' intention to use technology: A Turkish study. Campus-Wide Information Systems, 28(2), 93-101. Retrieved from: https://doi.org/10.1108/10650741111117798

Thompson, R. L., Higgins, C. A., \& Howell, J. M. (1991). Personal computing: toward a conceptual model of utilization. MIS Quarterly, 125-143. 
Traxler, J. (2009). Learning in a Mobile Age. International Journal of Mobile and Blended Learning (IJMBL), 1(1), 1-12. Retrieved from: https://doi.org/10.4018/jmbl.2009010101

Vallerand, R. J. (1997). Toward A Hierarchical Model of Intrinsic and Extrinsic Motivation. Advances in Experimental Social Psychology, 29(C), 271-360. Retrieved from: https://doi.org/10.1016/S00652601(08)60019-2

Venkatesh, V., \& Bala, H. (2008). Technology acceptance model 3 and a research agenda on interventions. Decision Sciences, 39(2), 273-315. Retrieved from: https://doi.org/10.1111/j.15405915.2008.00192.x

Venkatesh, V., \& Davis, F. D. (2000). A theoretical extension of the technology acceptance model: Four longitudinal field studies. Management Science, 46(2), 186-204. Retrieved from: https://doi.org/https://doi.org/10.1287/mnsc.46.2.186.11926

Venkatesh, V., Morris, M. G., Davis, G. B., \& Davis, F. D. (2003). User Acceptance of Information Technology: Toward a Unified View. Source: MIS Quarterly, 27(3), 425-478. Retrieved from: https://doi.org/10.2307/30036540

Wong, K. T., Teo, T., \& Russo, S. (2012). Influence of gender and computer teaching efficacy on computer acceptance among Malaysian student teachers: An extended technology acceptance model. Australasian Journal of Educational Technology, 28(7), 1190-1207. Retrieved from: https://ajet.org.au/index.php/AJET/article/view/796/96

Yu, Z. (2020). Visualizing co-citations of technology acceptance models in education. Journal of Information Technology Research, 13(1), 77-95. Retrieved from: https://doi.org/10.4018/JITR.2020010106 